\title{
Unilateral Conditioning Contractions Enhance Power Output in Elite Short Track Speed Skaters
}

(ㅇ)( $\odot \ominus$

Authors

Philippe Gimenez ${ }^{1,2}$, Evelyne Chicoine ${ }^{2}$, David Amarantini' ${ }^{3}$ Fabien Dal Maso4, Jonathan Tremblay ${ }^{2,5}$

\section{Affiliations}

1 Laboratoire C3S (EA 4660), Université de BourgogneFranche-Comté, Besançon, France

2 École de kinésiologie et des sciences de l'activité physique, Université de Montréal, Montréal, Canada

3 ToNIC, Toulouse Neurolmaging Center, Université de Toulouse, Inserm, UPS, France

4 École de kinésiologie et des sciences de l'activité physique, laboratoire de simulation et modélisation du mouvement, Université de Montréal, Montréal, Canada

5 Institut National du Sport du Québec, Montréal, Canada

\section{Key words}

exercise, post activation potentiation, fatigue, power

$\begin{array}{lr}\text { received } & 18.01 .2018 \\ \text { revised } & 16.10 .2018 \\ \text { accepted } & 19.10 .2018\end{array}$

\section{Bibliography}

DOI https://doi.org/10.1055/a-0770-4699

Sports Medicine International Open 2018; 2: E185-E190

(c) Georg Thieme Verlag KG Stuttgart · New York

ISSN 2367-1890

\section{Correspondence}

Dr. Philippe Gimenez

Laboratoire C3S (EA 4660), Université de

Bourgogne-Franche-Comté, Besançon, France
UPFR des Sports

31 Chemin de l'Epitaphe

25000 Besançon

France

Tel.: + 33/3/6308 25 53, Fax: + 33/3/8166 6836

philippe.gimenez@univ-fcomte.fr

\begin{abstract}
The objective of the present study was to assess the effect of unilateral lower-body-conditioning muscle contractions during multiple sets of fatiguing repeated jumps in elite athletes. Five elite short-track speed-skating athletes performed 9 sets of 6 maximal consecutive jumps on 2 separate occasions: with (COND) and without (CTRL) preliminary voluntary conditioning contractions (CC) 5 min before the beginning of the sets. The CC consisted of 2 consecutive $3 \mathrm{~s}$ maximal unilateral isometric squats against a fixed bar, resulting in a $6 \mathrm{~s}$ overall isometric contraction per leg. For each set, power output (PO) was measured using a linear position transducer and averaged over the 6 corresponding repeated jumps. The results showed that PO was significantly greater during the test in COND than in CTRL $(p<0.01)$. PO significantly decreased with sets, by $19.4 \pm 4.7$ and $15.2 \pm 7.6 \%(p<0.001)$ between the first and last set in COND and CTRL, respectively. A $2 \times 3$ s maximal unilateral isometric CC, performed $5 \mathrm{~min}$ before unloaded repeated jumps, significantly increased mean PO. These results suggest that unilateral conditioning contractions can enhance performance in subsequent bilateral repeated jumps.
\end{abstract}

\section{Introduction}

Short track speed skating is characterized by a succession of explosive efforts where performance is strongly correlated with the strength characteristics of the leg extensors of the athletes [11]. In this sport, the ability of an athlete to produce high power and accelerate at the start of the race can significantly affect the rank position entering the first turn, which is a discriminating factor of the race outcome on short distances [18]. The development of training or conditioning methods to enhance explosive muscular power of short track speed skaters, especially in the early phase of the race, could thus positively impact race outcomes.

Earlier studies have shown that the performance of a muscle is affected, at least in part, by its contractile history ("conditioning contractions", CC) that can induce post-activation potentiation (PAP) [35, 38]. PAP is known to be a phenomenon by which the performance of a muscle or muscle group, during explosive movements patterns requiring maximal strength and power, is enhanced 
by its prior contractile activity [38]. Fatigue and PAP resulting from CC can coexist [31], but optimal performance following CC occurs when positive effects of PAP offset the negative effects induced by fatigue $[22,35,38]$.

Previous results examining the application of PAP to enhance performance in acute voluntary contractions report conflicting findings with regard to its effects on performance [6, 9, 38, 40, 41]. Many factors have been shown to influence the effects of PAP, namely the time interval between the $\mathrm{CC}$ and exercise performance, the type of muscle fibres mainly recruited, the mode and intensity of the CC, the characteristics of the subsequent exercise and the training status of the subjects considered [8, 22,34]. For example, elite athletes show a greater improvement in explosive performance than recreationally trained subjects, probably due to a higher proportion of fast-twitch fibres $[6,9,16]$. High-intensity CC, mostly recruiting fast-twitch fibers, appear to be more effective in activating the mechanisms of PAP $[13,35]$.

Although the time interval between CC and the following exercise appears to be critical to maximize performance [8, 34, 38], little is known about the duration of the potential beneficial effects of PAP during long-lasting, fatiguing and repeated explosive exercises. While previous studies have reported the effects of PAP on a subsequent acute exercise consisting of a single jump, a set of consecutive jumps or a short cycle sprint $[7,10,12,13,40]$, no study focused on the effects of PAP on multiple sets of consecutive jumps. Indeed, investigating explosive exercises over multiples sets would be helpful to better understand the lasting effects of PAP and its practical outcomes in sports like short track speed skating where the performance is characterised by the repetition of explosive efforts.

The present study aimed to assess the effect of a lower body CC on subsequent repetitive sets of fatiguing maximum repeated jumps in elite short-track speed-skating athletes. The objective was twofold: first determine the effect of CC on peak power output (PO) produced during vertical repeated jumps and second, how this effect is sustained over sets of repeated vertical jumps. It was hypothesized that prior lower-body CC would result in a higher mechanical power, especially during the first sets of repetitive maximum jumps, and that this potentiating effect would disappear in the subsequent sets.

\section{Materials and Methods}

\section{Subjects}

Five elite short-track speed-skating athletes volunteered for participation in the study ( 3 men and 2 women, age: $20.2 \pm 3.1$ years, height: $1.71 \pm 0.10 \mathrm{~m}$, mass: $67.6 \pm 11.6 \mathrm{~kg}$; mean $\pm S D$ ). All participants were training at the Montreal National Training Center and were competing in national and international competitions. Before participation, written informed consent was obtained from each adult athlete and from the parents of each minor athlete. The protocol was in accordance with procedures approved by the University of Montreal ethics committee in health research (certificate number: 13-061-CERES-D) and meets the ethical standards of Sports Medicine International Open [17].

\section{Testing protocol}

The testing protocol consisted of 9 sets of 6 maximal consecutive jumps with sets interspaced by a $5 \mathrm{~s}$ unweighted isometric squat with the trunk and knees flexed at $45^{\circ}$ and $90^{\circ}$, respectively. Over a $1000 \mathrm{~m}$ race, skaters typically perform 9 laps on a $111.12 \mathrm{~m}$ oval track with $\sim 12$ strides ( $~ 6$ per turn) and $\sim 2$ gliding phases per lap. Each set of 6 jumps were initiated from an isometric squat position, with the trunk and knees flexed at $45^{\circ}$ and $90^{\circ}$ respectively. The 6 jumps were performed consecutively with no time interval between them (which involves a stretch shortening cycle for each jump preceded by another one.)

Two weeks before the test sessions, subjects performed a familiarization session with the testing protocol, 5 min after having completed the same directed 5-min dynamic warm-up, including stationary jogging, high-knees, and half-squat exercises. Each subject randomly performed the testing protocol (see $>$ Fig. 1) on 2 separate occasions (one week apart): with (experimental condition: COND) and without (control condition: CTRL) preliminary voluntary CC 5 min before the execution of the 9 sets. The participants were instructed to keep their hands on their hips and to jump as high as possible throughout the testing protocol. Verbal encouragement and verbal cues to correct knee or trunk positions were also provided as needed.

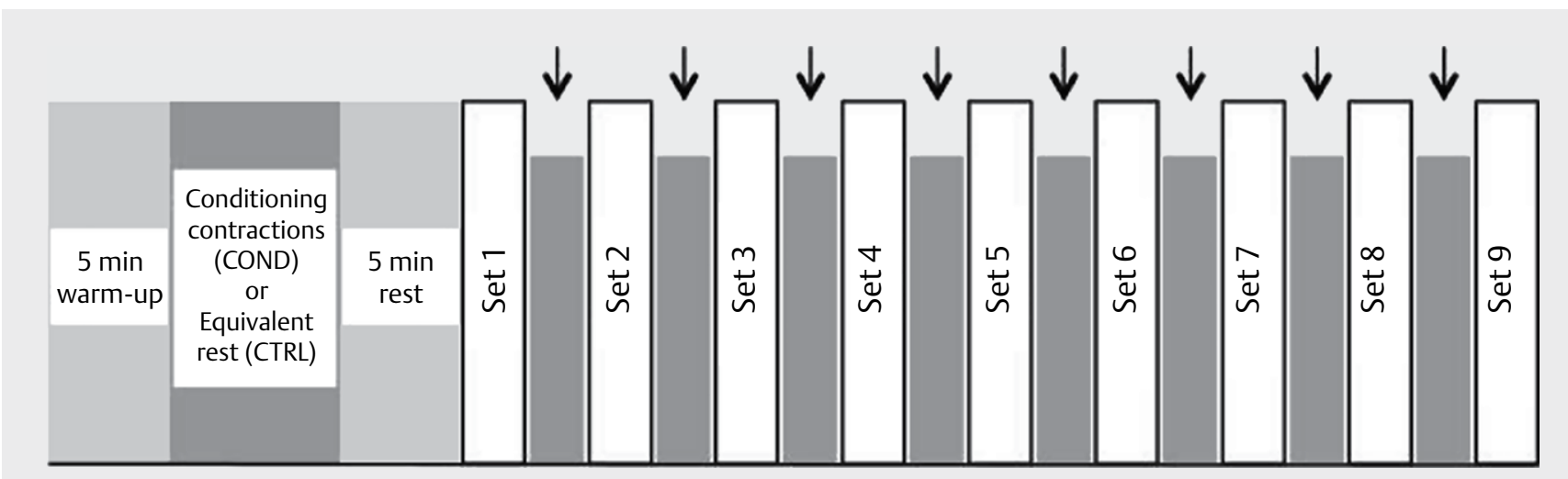

- Fig. 1 Schematic representation of the experimental protocol. The CC were performed only in COND, rest of equivalent duration was given in CTRL. Each set comprised 6 maximal unweighted repeated jumps immediately followed by a $5 \mathrm{~s}$ isometric squat (black arrow). 


\section{Conditioning contractions}

The CC consisted of two $3 \mathrm{~s}$ maximal unilateral isometric squats against a fixed barbell, for each leg and alternately, resulting in $6 \mathrm{~s}$ of overall CC for each leg. Each leg was thus alternately and maximally solicited, twice with about $5 \mathrm{~s}$ rest between repetitions in order to switch leg.

\section{Measurements}

For each jump, we determined the average power output (PO) using the average velocity during the push-off phase with a linear position transducer [14] (Tendo Sports Machine, Trencin, Slovakia) extending a string to a belt fastened around the hips of the participants. We positioned the linear position transducer on the ground between the subject's feet and measured the linear displacement and time during the execution of the jumps. The mean PO was computed and normalized to body mass for each of the 6 repeated jumps.

\section{Statistical analyses}

Data are presented as mean \pm SD unless otherwise specified. A linear mixed model analysis performed using R [30] (R Core Team (2014), R: A language and environment for statistical computing. R Foundation for Statistical Computing, Vienna, Austria) and the Ime4 [2] package was applied with the factors Condition (COND and CTRL) and Set (1-9) to determine if there were significant differences between different time points and treatment modalities. Residual analysis indicated that the model satisfied the normality assumption required for the linear mixed model. Tukey's post-hoc analysis was used to identify specific sets where PO was different. The level of significance was set at $\alpha=0.05$. Effect size was estimated by computing partial eta squared $\left(\eta_{p}{ }^{2}\right)$, with $0.01,0.13$ and 0.26 representing a small, medium and large effect, respectively [29].

\section{Results}

The statistical analysis revealed significant sets $\left(p<0.001 ; \eta_{p}{ }^{2}=0.93\right)$, and conditions effects $\left(p<0.01 ; \eta_{p}{ }^{2}=0.73\right)$, whereas the interaction effect was not significant $(p=0.45)$. Mean and individual changes in PO for COND and CTRL conditions are shown in > Fig. 2, > 3, respectively. PO significantly decreased with sets, by $19.4 \pm 4.7 \%$ (from $15.0 \pm 1.9$ to $12.1 \pm 1.5 \mathrm{~W} / \mathrm{kg}: \mathrm{p}<0.001$ ) and $15.2 \pm 7.6 \%$ (from $14.3 \pm 2.0$ to $12.1 \pm 1.3 \mathrm{~W} / \mathrm{kg}: \mathrm{p}<0.001$ ), between the first and last set in COND and CTRL, respectively. Overall, $P O$ was greater in COND than in CTRL ( $13.7 \pm 0.70$ and $13.2 \pm 0.69 \mathrm{~W} / \mathrm{kg}$ respectively, $\mathrm{p}<0.01)$, which corresponds to an increase of $3.77 \pm 3.82 \%$ in COND compared to CTRL during the whole sets. Qualitatively, > Fig. 3 shows that for all the participants, the PO was greater in the COND than the CTRL condition during the 3 first blocks. During the subsequent blocks, the PO was greater in the COND than in the CTRL condition in $73 \%$ of the cases.

\section{Discussion}

The present study investigated the effects of lower-body maximal unilateral isometric CC $(2 \times 3 \mathrm{~s}$, total of $6 \mathrm{~s}$ for each leg $)$, on the average $\mathrm{PO}$ in 9 sets of 6 consecutive jumps interspersed with iso- metric squats ( $5 \mathrm{~s}$ ) in elite short-track speed-skating athletes. Our main finding was that maximal unilateral isometric CC lead to a significant increase of PO.

Previous studies required participants to perform several sets of exercises after a CC, but participants were allowed to rest between sets. This was done to investigate the optimum timing of CC to maximize potentiation [37], which was shown to have a large interindividual variability [4]. For example, Kilduff et al. [24] showed that the optimal recovery time to maximize the effect of PAP on countermovement jump performance was $8 \mathrm{~min}$ in professional rugby players. More recently, Bogdanis et al. [4] had national-level track and field athletes perform 10 countermovement jumps following either isometric, eccentric or concentric CC with varying recovery durations (from $15 \mathrm{~s}$ up to $21 \mathrm{~min}$ ). These authors reported a very high intersubject variability in the optimal recovery time, which partly depended on the type of contraction. However, after the isometric CC, all subjects reached their peak performance between 2 and $8 \mathrm{~min}, 80 \%$ between 4 and $6 \mathrm{~min}$, whereas $50 \%$ of them performed best after 4 min of recovery time. Thus, in the present study, we chose 5 min as a potential optimal recovery time between the CC and the $1^{\text {st }}$ set of repeated jumps. As in track and field athletes [4], this delay was appropriate to increase the PO produced during the present 9 sets of 6 maximal consecutive jumps protocol in short-track speed-skating athletes.

The amplitude and direction of the observed enhancement of performance could be, at least partly, explained by the training experience of the short track speed skaters involved in the current study. Indeed, previous research evidenced that trained subjects may show greater PAP responses than untrained individuals $[6,33]$. For example, Chiu et al. [6] examined the effects of a pre-activation exercise ( 5 sets of parallel back squats at $90 \%$ of 1 RM, with 2 min rest between sets) on 2 consecutive sets of squat jumps (rebound or concentric-only squat jumps) with various loads. There was a 1 min rest period between each load and an additional 10 min between the 2 sets. The authors reported that the heavy resistance CC elicited a significant increase in performance, but only when the

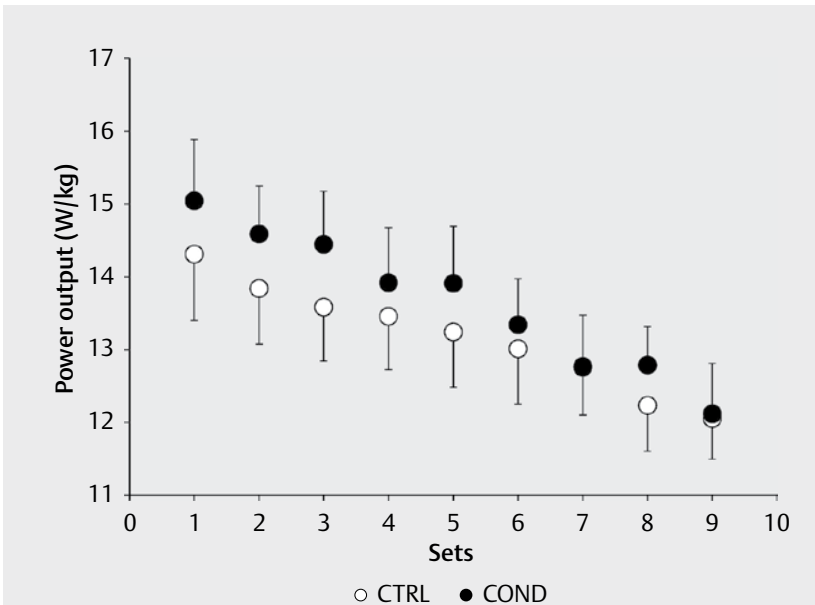

- Fig. 2 Mean and SE of average power output in CTRL (open circles) and in COND (black circles) over the 9 consecutive sets of repeated jumps. 


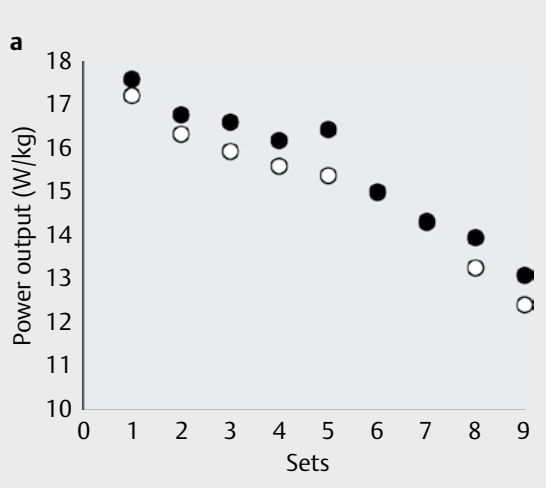

d 18

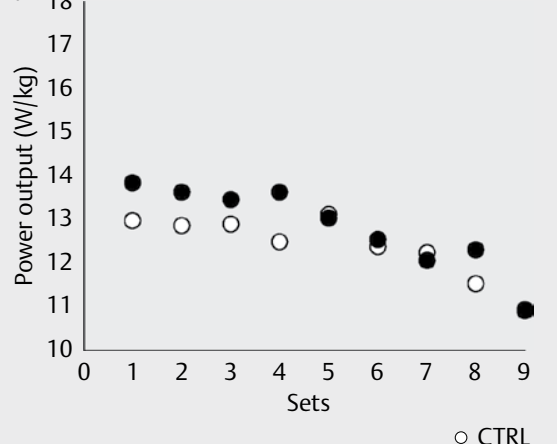

b

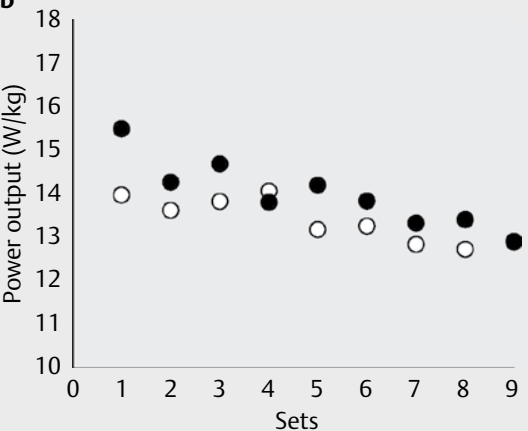

c

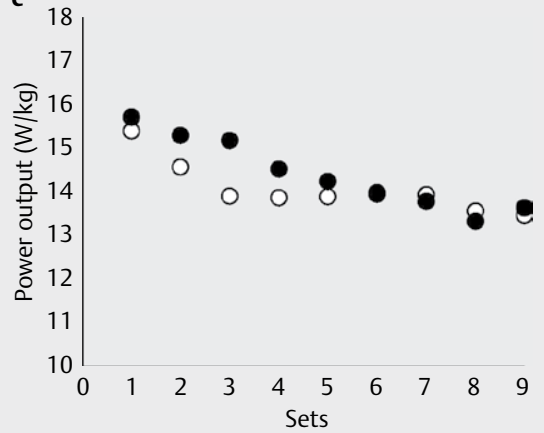

e 18

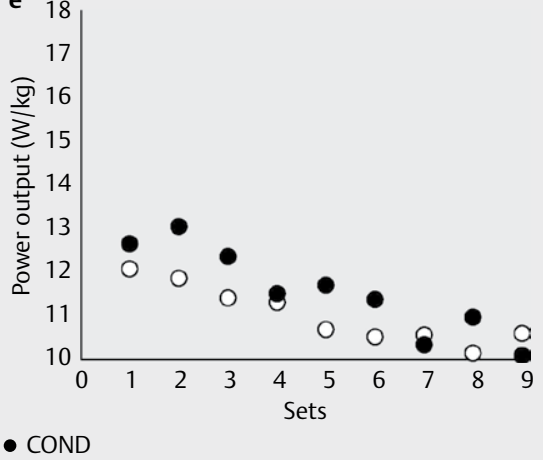

- Fig. 3 Individual power output in CTRL (open circles) and in COND (black circles) over the 9 consecutive sets of repeated jumps (subjects a, b, c, $\mathbf{d}$ and $\mathbf{e}$ ).

sample was split into 2 groups based on training experience, with a significant positive impact only observed in the trained subjects. The training experience of the short track speed skaters involved in the current study could thus explain the increase in performance observed.

Furthermore, studies have so far reported conflicting results when using dynamic $[26,38,41]$ or maximal isometric contractions $[15,33]$. This discrepancy could be due to the volume of CC, however Bogdanis et al. [4] compared an equivalent volume of different types of CC and found that both isometric and eccentric contractions lead to higher countermovement jump performance in comparison to a control condition (without CC), whereas concentric contractions had no impact. Moreover, compared to the baseline value (best countermovement jump performance determined after a standardized warm-up in each condition), performance was improved only in the isometric condition. Our results are in line with these observations and corroborate those of Miyamoto et al. [27], who reported that $\sim 5$-s isometric CC can enhance subsequent dynamic voluntary performance.

Although somewhat speculative, 2 arguments can be raised to support the use of a conditioning protocol involving CC performed separately and alternately on each leg to improve performance in both single and repetitive explosive efforts. Firstly, the reduction in the base of support induced by unilateral isometric CC could potentially increase the solicitation of hip muscle stabilizers [5, 25]. This may lead to an improvement of performance through greater acti- vation of hip stabilizers and improved transmission of forces of the primary power producers. Secondly, improvement in repeated jump performance following a unilateral CC protocol may be related to the fact that maximum voluntary force produced by a subject with the muscles of one limb is less when these muscles are active simultaneously with the homologous muscles in the contralateral limb than when they are active alone [3]. With regards to previous studies on the bilateral deficit [32, 39], it may be hypothesized that unilateral CC could have lead a greater activation of the targeted motor units contributing to the increase in the average power output. However, further studies should be conducted to draw definite conclusions about these 2 hypotheses.

The small sample size $(n=5)$ in the current study is a limitation, as for investigations with elite competitors of less populated sports such as rowing (Jensen et al. [23]; $n=7$ ), biathlon (Hesford et al. [20]; $n=2$ ), alpine skiing (Barelle et al. [1]; $n=4$ ), and short track speed skating (e.g., Haug et al. [19]; $n=5$, Hesford et al. [21]; $n=6$ ). But our results suggest that $2 \times 3$ s maximal unilateral isometric CC executed by speed skaters, lead to a significant increase in bilateral power output, in a subsequent exercise performed 5 min later, at least for a few seconds. Another limitation is that the exercise did not represent a real speed skating condition, with a force application mainly directed vertically whereas in speed skating the force application is directed more horizontally. A high PO measured vertically associated with a high quality of vertical-to-horizontal transfer can lead to high maximal horizontal forces that is a primordial for short sprint 
acceleration performances [28]. However, in the present study we hypothesized that the CC did not affect the quality of vertical-to-horizontal transfer of force, indicating that the observed increase in PO could result in an improvement of sprint-acceleration performances during speed skating. Even though further studies should be conducted to draw definite conclusions in view of lower limbs' force-velocity-power properties [36] during real speed-skating conditions, athletes in various sports could benefit from this enhancement of performance, especially if it can help to achieve a strategic lead at the beginning of a race, as is the case in short track speed skating [18].

The present study showed that a maximal unilateral isometric CC induced an increase in mean power output during repetitive sets of vertical squat jumps. Such an improvement could be beneficial in sports involving repetitive powerful efforts, such as encountered in short-track speed-skating competition.

\section{Acknowledgements}

The authors would like to thank the athletes for their participation and the coaching staff from the Montreal National Short Track Speed Skating Training Center for collaborating on the elaboration of the experimental protocol. The authors also wish to acknowledge the statistical advice of Robin Baurès (Brain and Cognition Research Center (CerCo), UMR 5549 CNRS/Paul Sabatier University, Toulouse, France).

\section{Conflict of Interest}

No conflict of interest has been declared by the authors.

\section{References}

[1] Barelle C, Ruby A, Tavernier M. Original research experimental model of the aerodynamic drag coefficient in Alpine skiing. J Appl Biomech 2004; 20: 167-176

[2] Bates D, Maechler M, Bolker B, Walker S. Ime4: Linear mixed-effects models using eigen and S4. R package version 1.1-7 http://CRAN.Rproject.org/package $=$ Ime4 $\mathrm{R}$ Packag version 2014

[3] Bobbert MF, de Graaf WW, Jonk JN, Casius LJR. Explanation of the bilateral deficit in human vertical squat jumping. J Appl Physiol 2006; 100: 493-499

[4] Bogdanis GC, Tsoukos A, Veligekas P, Tsolakis C, Terzis G. Effects of muscle action type with equal impulse of conditioning activity on post activation potentiation. J Strength Cond Res 2014; 1-31

[5] Boudreau SN, Dwyer MK, Mattacola CG, Lattermann C, Uhl TL, McKeon JM. Hip-muscle activation during the lunge, single-leg squat, and step-up-and-over exercises. J Sport Rehabil 2009; 18: 91-103

[6] Chiu LZF, Fry AC, Weiss LW, Schilling BK, Brown LE, Smith SL. Postactivation potentiation response in athletic and recreationally trained individuals. J Strength Cond Res 2003; 17: 671-677

[7] Crow JF, Buttifant D, Kearny SG, Hrysomallis C. Low load exercises targeting the gluteal muscle group acutely enhance explosive power output in elite athletes. J Strength Cond Res 2012; 26: 438-442

[8] Docherty D, Hodgson M]. The application of postactivation potentiation to elite sport. Int J Sports Physiol Perform 2007; 2: 439-444
[9] Duthie GM, Young WB, Aitken DA. The acute effects of heavy loads on jump squat performance: An evaluation of the complex and contrast methods of power development. J Strength Cond Res 2002; 16: 530-538

[10] Esformes ], Bampouras T. Effects of back squat depth on lower-body postactivation potentiation. J Strength Cond Res 2013; 27: 2997-3000

[11] Felser S, Behrens M, Fischer S, Heise S, Bäumler M, Salomon R, Bruhn S. Relationship between strength qualities and short track speed skating performance in young athletes. Scand J Med Sci Sports 2016; 26: 165-171

[12] French DN, Kraemer W], Cooke CB. Changes in dynamic exercise performance following a sequence of preconditioning isometric muscle actions. J Strength Cond Res 2003; 17: 678-685

[13] Fukutani A, Takei S, Hirata K, Miyamoto N, Kanehisa H, Kawakami Y. Influence of the intensity of squat exercises on the subsequent jump performance. J Strength Cond Res 2014; 28: 2236-2243

[14] Garnacho-Castaño MV, López-Lastra S, Maté-Muñoz JL. Reliability and validity assessment of a linear position transducer. J Sport Sci Med 2014; 14: 128-136

[15] Gossen ER, Sale DG. Effect of postactivation potentiation on dynamic knee extension performance. Eur J Appl Physiol 2000; 83: 524-530

[16] Hamada T, Sale DG, MacDougall JD, Tarnopolsky MA. Postactivation potentiation, fiber type, and twitch contraction time in human knee extensor muscles. J Appl Physiol 2000; 88: 2131-2137

[17] Harriss D, Macsween A, Atkinson G. Standards for ethics in sport and exercise science research: 2018 update. Int J Sports Med 2017; 38: 1126-1131

[18] Haug WB, Drinkwater E], Mitchell L], Chapman DW. The relationship between start performance and race outcome in elite $500-\mathrm{m}$ short-track speed skating. Int J Sports Physiol Perform 2015; 10 : 902-906

[19] Haug W, Spratford W, Williams K, Chapman D, Drinkwater E. Differences in end range of motion vertical jump kinetic and kinematic strategies between trained weightlifters and elite short track speed skaters. J Strength Cond Res 2015; 29: 2488-2496

[20] Hesford CM, Laing S, Cooper CE. Using portable NIRS to compare arm and leg muscle oxygenation during roller skiing in biathletes: A case study. Adv Exp Med Biol 2013; 789: 179-184

[21] Hesford CM, Laing S], Cardinale M, Cooper CE. Asymmetry of quadriceps muscle oxygenation during elite short-track speed skating. Med Sci Sports Exerc 2012; 44: 501-508

[22] Hodgson M, Docherty D, Robbins D. Post-activation potentiation: Underlying physiology and implications for motor performance. Sport Med 2005; 35: 585-595

[23] Jensen AM. The use of neuro emotional technique with competitive rowers: A case series. J Chiropr Med 2011; 10: 111-117

[24] Kilduff LP, Owen N, Bevan H, Bennett M, Kingsley MIC, Cunningham D. Influence of recovery time on post-activation potentiation in professional rugby players. J Sport Sci 2008; 26: 795-802

[25] Krause DA, Jacobs RS, Pilger KE, Sather BR, Sibunka SP, Hollman JH. Electromyographic analysis of the gluteus medius in five weight-bearing exercises. J Strength Cond Res 2009; 23: 2689

[26] Mangus BC, Takahashi M, Mercer JA, Holcomb WR, McWhorter JW, Sanchez R. Investigation of vertical jump performance after completing heavy squat exercises. J Strength Cond Res 2006; 20: 597-600

[27] Miyamoto N, Kanehisa H, Kawakami Y. Potentiation of maximal voluntary concentric torque in human quadriceps femoris. Med Sci Sports Exerc 2012; 44: 1738-1746

[28] Morin JB, Samozino P. Interpreting power-force-velocity profiles for individualized and specific training. Int J Sports Physiol Perform 2016; 11: $267-272$ 
[29] Perdices M. Null hypothesis significance testing, p-values, effects sizes and confidence intervals. Brain Impair 2018; 19: 70-80

[30] Pinheiro J, Bates D, DebRoy S, Sarkar D.Team RC. nlme: Linear and Nonlinear Mixed Effects Models. R Packag version 3, 1-118, 2014

[31] Rassier DE, MacIntosh BR. Coexistence of potentiation and fatigue in skeletal muscle. Brazilian J Med Biol Res 2000; 33: 499-508

[32] Rejc E, Lazzer S, Antonutto G, Isola M, Di Prampero PE. Bilateral deficit and EMG activity during explosive lower limb contractions against different overloads. Eur J Appl Physiol 2010; 108: 157-165

[33] Rixon KP, Lamont HS, Bemben MG. Influence of type of muscle contraction, gender, and lifting experience on postactivation potentiation performance. J Strength Cond Res 2007; 21: 500-505

[34] Robbins DW. Postactivation potentiation and its practical applicability: A brief review. J Strength Cond Res 2005; 19: 453-458

[35] Sale DG. Postactivation potentiation: Role in human performance. Exerc Sport Sci Rev 2002; 30: 138-143

[36] Samozino P, Rabita G, Dorel S, Slawinski J, Peyrot N, Saez de Villarreal E, Morin JB. A simple method for measuring power, force, velocity properties, and mechanical effectiveness in sprint running. Scand J Med Sci Sport 2016; 26: 648-658
[37] Seitz LB, de Villarreal ES, Haff GG. The temporal profile of postactivation potentiation is related to strength level. J Strength Cond Res 2014; 28: 706-715

[38] Tillin NA, Bishop D. Factors modulating post-activation potentiation and its effect on performance of subsequent explosive activities. Sport Med 2009; 39: 147-166

[39] Vandervoort AA, Sale DG, Moroz J. Comparison of motor unit activation during unilateral and bilateral leg extension. J Appl Physiol 1984; 56: 46-51

[40] Weber KR, Brown LE, Coburn JW, Zinder SM. Acute effects of heavy-load squats on consecutive squat jump performance. J Strength Cond Res 2008; 22: 726-730

[41] Young W. Acute enhancement of power performance from heavy load squats. J strength Cond Res 1998; 12: 82-84 